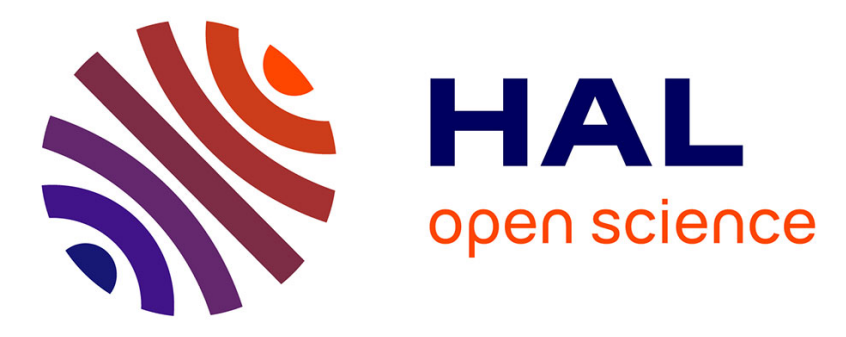

\title{
Reduced brain amyloid burden in elderly patients with narcolepsy type 1
}

Audrey Gabelle, Isabelle Jaussent, Fayçal Ben Bouallègue, Sylvain Lehmann, Régis Lopez, Lucie Barateau, Caroline Grasselli, Carole Pesenti, Delphine de Verbizier, Severine Beziat, et al.

\section{To cite this version:}

Audrey Gabelle, Isabelle Jaussent, Fayçal Ben Bouallègue, Sylvain Lehmann, Régis Lopez, et al.. Reduced brain amyloid burden in elderly patients with narcolepsy type 1. Annals of Neurology, In press, 10.1002/ana.25373 . hal-01911698

\section{HAL Id: hal-01911698 \\ https://hal.science/hal-01911698}

Submitted on 25 May 2020

HAL is a multi-disciplinary open access archive for the deposit and dissemination of scientific research documents, whether they are published or not. The documents may come from teaching and research institutions in France or abroad, or from public or private research centers.
L'archive ouverte pluridisciplinaire HAL, est destinée au dépôt et à la diffusion de documents scientifiques de niveau recherche, publiés ou non, émanant des établissements d'enseignement et de recherche français ou étrangers, des laboratoires publics ou privés. 


\title{
Reduced Brain Amyloid Burden in Elderly Patients with Narcolepsy Type 1
}

\author{
Audrey Gabelle, MD, ${ }^{1,2,3}$ Isabelle Jaussent, MD, ${ }^{2,3}$ Fayçal Ben Bouallègue, MD, ${ }^{2,4,5}$ \\ Sylvain Lehmann, MD, 2,6 Régis Lopez, MD, 2,3,7 Lucie Barateau, MD, 2,3,7 \\ Caroline Grasselli, MSc, ${ }^{1}$ Carole Pesenti, MSc, ${ }^{7}$ Delphine de Verbizier, MD, ${ }^{4}$ \\ Séverine Béziat, MSc, ${ }_{1}^{2,3}$ Denis Mariano-Goulart, MD, ${ }^{2,4,5}$ Bertrand Carlander, MD, \\ Yves Dauvilliers, MD, 2,3,7 and the Alzheimer's Disease Neuroimaging Initiative and \\ Multi-Domain Intervention Alzheimer's Prevention Trial study groups
}

Objective: To determine whether brain amyloid burden in elderly patients with narcolepsy type 1 (NT1) is lower than in controls, and to assess in patients with NT1 the relationships between amyloid burden, cerebral spinal fluid (CSF) markers of Alzheimer disease (AD), CSF orexin-A, and cognitive profile.

Methods: Cognitive and ${ }^{18} \mathrm{~F}$-florbetapir positron emission tomography (PET) data were compared in patients with NT1 aged $\geq 65$ years $(n=23)$ and in age- and sex-matched controls free of clinical dementia selected from the Alzheimer's Disease Neuroimaging Initiative (ADNI; $n=69$ ) and the Multi-Domain Intervention Alzheimer's Prevention Trial (MAPT18F AV45-PET; $n=23$ ) cohorts. The standardized uptake values (SUVs) of the cortical retention index for 6 regions of interest were computed and averaged to create a mean SUV ratio normalized to 3 subcortical reference regions (cerebellum, pons, and a composite region). A cortical/cerebellum SUV ratio $\geq 1.17$ defined positive PET amyloid.

Results: Lower cortical amyloid burden was observed in the NT1 than in the ADNI and MAPT-AV45 groups (mean cortical/cerebellum SUV ratios $=0.95 \pm 0.15,1.11 \pm 0.18[p<0.0001]$, and $1.14 \pm 0.17[p=0.0005]$, respectively). Similar results were obtained with all subcortical reference regions and for all cortical regions of interest, except cingulum. Only 1 patient with NT1 (4.4\%) had positive PET amyloid compared with $27.5 \%$ in the ADNI and $30.4 \%$ in the MAPT-AV45 group. In the NT1 group, cortical or regional amyloid load was not associated with CSF orexin-A, CSF AD biomarkers, or neuropsychological profile.

Interpretation: Lower brain amyloid burden, assessed by ${ }^{18} \mathrm{~F}$-florbetapir PET, in patients with NT1 suggests delayed appearance of amyloid plaques.

ositron emission tomographic (PET) imaging with tracers that bind specifically to amyloid- $\beta(A \beta)$ aggregates can be used to quantify the accumulation of amyloid plaques, many years before the clinical symptoms of Alzheimer disease (AD). ${ }^{1,2}$ The dynamics of $A \beta$ changes and the large variability in progressive cognitive decline remain mostly unclear in subjects at risk for $\mathrm{AD} .{ }^{3,4}$ Recent findings showed interactions between $A \beta$ peptides and sleep/wake patterns with efficient convective clearance of $A \beta$ during deep sleep and $A \beta$ accumulation in brain interstitial fluid during wakefulness. ${ }^{5-7}$ Increased neuronal activity during wakefulness might promote AD development, with a strong relationship with a wake-promoting peptide named orexin-A/hypocretin-1. ${ }^{8}$ Specifically, in

Address correspondence to Dr Dauvilliers, Narcolepsy National Reference Center, Sleep Center, Department of Neurology, CHU Montpellier, University of Montpellier, Montpellier, France. E-mail: ydauvilliers@yahoo.fr

From the ${ }^{1}$ Memory Research and Resources Center, Department of Neurology, Gui de Chauliac University Hospital Center; ${ }^{2}$ University of Montpellier; ${ }^{3}$ National Institute of Health and Medical Research U1061, Neuropsychiatry: Epidemiological and Clinical Research; ${ }^{4}$ Department of Nuclear Medicine, Montpellier University Hospital; ${ }^{5}$ PhyMedExp, National Institute of Health and Medical Research, National Center for Scientific Research; ${ }^{6}$ National Institute of Health and Medical Research U1183, Saint Eloi Hospital; and ${ }^{7}$ Narcolepsy National Reference Center, Sleep Center, Department of Neurology, Montpellier University Hospital Center, Montpellier, France. 
transgenic mice that overexpress amyloid precursor protein (APP), $A \beta$ level increases during wakefulness and after orexinA infusion and decreases during sleep and after infusion of an orexin-A receptor antagonist. ${ }^{8}$ In APP/presenilin 1 transgenic mice in which the orexin gene also is knocked out, $A \beta$ brain load is decreased and sleep time is increased. ${ }^{9}$ Moreover, sleep deprivation by rescue of orexinergic neurons in these mice increases the amount of brain $A \beta .{ }^{9}$

Conversely, results on orexin-A levels in cerebrospinal fluid (CSF) samples from patients with $\mathrm{AD}$ are conflicting. In a postmortem analysis, the number of orexin neurons in the hypothalamus and the concentration of orexin in ventricular CSF were reduced in patients with $\mathrm{AD}$ compared with controls. ${ }^{10}$ However, higher CSF orexin-A levels were observed in patients with $\mathrm{AD}$ compared with controls. ${ }^{11-13}$ Moreover, in one study, CSF orexin-A levels were correlated with tau protein levels and sleep-wake alterations in patients with $\mathrm{AD} .{ }^{13}$ Recently, an experimental study showed that suppression of slow-wave sleep increased CSF A $\beta$ concentrations the following day. ${ }^{14}$ Altogether, these data suggest that sleep-wake cycle abnormalities and orexin levels may influence amyloid clearance and brain amyloidosis.

Narcolepsy type 1 (NT1) is an orphan chronic disease characterized by excessive daytime sleepiness (EDS) and cataplexy, and caused by the destruction of orexin neurons. ${ }^{15,16}$ It represents an interesting model to improve our understanding of the relationship between $A \beta$ and the orexin pathways. NT1 typically starts in the teens or twenties. ${ }^{17}$ Therefore, these patients could be partially protected from $\mathrm{AD}$, a disease that develops and progresses over several years prior to clinical symptoms. Only 1 postmortem study has addressed this hypothesis by analyzing the presence of neuropathological lesions consistent with $\mathrm{AD}$ in brain tissues from a few patients with narcolepsycataplexy. It reported that $33 \%$ of patients had $\mathrm{AD}$ lesions, a proportion similar to what would be expected in the general population. ${ }^{18}$ However, data on the narcolepsy phenotype, CSF orexin-A levels, and orexinergic neuron quantification were not available, except for 1 patient.

Therefore, to determine whether elderly patients with NT1 have a lower brain amyloid burden and lower $\mathrm{AD}$ risk compared with age- and sex-matched controls free of clinical dementia, we used brain PET with ${ }^{18} \mathrm{~F}$-florbetapir, which binds to $A \beta$ with high affinity and specificity. We also assessed in the NT1 group the relationships between $A \beta$ load, and CSF-AD markers, CSF orexin-A level, and cognitive profile.

\section{Subjects and Methods \\ Patients}

We recruited 23 consecutive patients with NT1 older than 65 years $(14$ men and 9 women; median age $=71$ years, range
65-86) at the Reference National Center for Narcolepsy of Montpellier, France. NT1 was diagnosed according to the ICSD-3 criteria $^{19}$ : EDS, clear-cut cataplexy, and mean sleep latency on the Multiple Sleep Latency Test $\leq 8$ minutes with $\geq$ 2 sleep onset rapid eye movement sleep periods and/or CSF orexin-A level $<110 \mathrm{pg} / \mathrm{ml}$ (in all 20 patients who underwent lumbar puncture). All patients had the human leukocyte antigen (HLA) DQB1*06:02 genotype.

A standardized face-to-face clinical examination and interview evaluated the body mass index, medical history, age at NT1 onset and at diagnosis, EDS severity with the Epworth Sleepiness Scale (ESS), ${ }^{20}$ cataplexy frequency, depressive symptoms using the Beck Depression Inventory scale, ${ }^{21}$ and health-related quality of life with the European Quality of Life Five Dimensions scale. $^{22}$ The use of stimulants and anticataplectic drugs was recorded. A semistructured interview of the patient and a reliable informant (eg, family member) focused on 6 domains of cognitive and functional performance (ie, Memory, Orientation, Judgment \& Problem Solving, Community Affairs, Home \& Hobbies, and Personal Care) was used to establish the 5-point Cognitive Dementia Rating (CDR) scale score (ie, $0=$ normal; $0.5=$ very mild dementia; $1=$ mild dementia; $2=$ moderate dementia; 3 = severe dementia). ${ }^{23}$ The comprehensive neuropsychological evaluation included the presence of memory complaint based on clinical interview, the Mini-Mental State Examination (MMSE), Free and Cued Selective Reminding Test (FCSRT; 16 items), a verbal fluency test, a counting span test, Trail Making Test A and B (TMT-A and B), Frontal Assessment Battery (FAB) test, Rey-Osterrieth Complex Figure Test, and praxis and motor planning assessment. All patients underwent ${ }^{18} \mathrm{~F}$-florbetapir PET brain imaging.

\section{Standard Protocol Approvals, Registrations, and Patient Consents}

All participants gave their written informed consent to participate in this study, which was approved by the Montpellier University Hospital ethics committee (www.dinicaltrials.gov NCT03378453).

\section{Controls}

Two age- and sex-matched control groups free of clinical dementia were selected from two different cohorts. The first group was selected from the Alzheimer's Disease Neuroimaging Initiative (ADNI) database (www.loni.ucla.edu/ADNI) after the scientific committee's agreement. Paired cases (3 controls for each patient with NT1) were defined as cognitively normal subjects or subjects with only subjective memory complaints $(\mathrm{CDR}=0$ or 0.5$)$, and with available brain ${ }^{18} \mathrm{~F}$-florbetapir PET data. Their MMSE score ranged from 24 to 30 , and the subjective memory complaint score was 16 or higher for the first 12 questions of the Cognitive Complaint Index. ${ }^{24}$ The second age- and sex-matched control sample was selected from the Multi-Domain Intervention Alzheimer's Prevention Trial (Florbetapir F 18 ((E)-4-(2-(6-(2-(2-(2-[18F] fluoroethoxy)ethoxy)ethoxy)pyridin-3-yl)vinyl)-N- methylbenzenamine also known as 18F-AV-45)) PET ancillary study cohort (www.clinicaltrials.gov NCT00672685). ${ }^{25}$ The selected participants (1 control for each patient with NT1) were defined as 
cognitively normal or with subjective memory complaint $(\mathrm{CDR}=0$ or 0.5$)$, and with available ${ }^{18} \mathrm{~F}$-florbetapir PET data. Neuropsychological evaluations included the MMSE, FCSRT-16, Wechsler Adult Intelligence Scale-Revised, and Category Naming Test.

\section{Main Study Outcome: Assessment of A $\beta$ Brain Deposition by ${ }^{18} \mathrm{~F}$-Florbetapir PET Imaging}

The PET image acquisition procedure was similar in the NT1 and MAPT-AV45 groups. Imaging was performed for 15 minutes at 50 minutes after injection of $370 \mathrm{MBq}{ }^{18} \mathrm{~F}$-florbetapir. Images were reconstructed using 3-dimensional ordered-subset expectation maximization ( 24 subsets, 12 iterations) followed by Gaussian postfiltering with $5 \mathrm{~mm}$ full width at half maximum (FWHM). As concurrent structural magnetic resonance imaging (MRI) data were not available for most patients, PET images were spatially normalized to the standard Montreal Neurological Institute (MNI) space by coregistration to a generic T1-weighted MRI template using SPM12. ${ }^{26}$

The ${ }^{18}$ F-florbetapir PET data for the ADNI group consisted of $4 \times 5$-minute frames acquired at 50 to 70 minutes after ${ }^{18} \mathrm{~F}$-florbetapir injection. They were realigned, averaged, resliced onto a common voxel size, and smoothened with an $8 \mathrm{~mm}$ FWHM Gaussian kernel. ${ }^{27}$ Structural MRI images acquired concurrently with the baseline ${ }^{18} \mathrm{~F}$-florbetapir PET images were used as a structural template to spatially normalize the PET images. Briefly, for each subject, the baseline ${ }^{18}$ F-florbetapir PET images were rigidly coregistered with the baseline structural T1-weighted MRI images by maximizing the normalized mutual information. The individual T1-weighted MRI images were nonlinearly coregistered to the standard MNI space MRI template using tissue probability maps delivered with SPM. Nonlinear transformation was then used to spatially normalize the coregistered PET images to the MNI space. To compare PET data with consistent resolutions, images of the NT1 cohort were postsmoothened using a proper Gaussian kernel to adjust their final resolution to that of postprocessed PET data for the ADNI group $(8 \mathrm{~mm})$. The final resolution of the available PET data for the MAPT-AV45 group was slightly lower $(9.5 \mathrm{~mm})$ due to systematic postsmoothening using SPM12.

For each subject, the ${ }^{18} \mathrm{~F}$-florbetapir PET voxels were labeled according to the maximum probability atlas derived from the MICCAI 2012 Grand Challenge and Workshop on MultiAtlas Labeling and provided by Neuromorphometrics (neuromorphometrics.com) under academic subscription. The standardized uptake values (SUVs) of the cortical retention index were computed in 6 cortical regions of interest (ie, frontal, parietal, temporal, precuneus, anterior, and posterior cingulate cortices) and were averaged to create a mean cortical SUV that defines the amyloid load. Cortical SUV ratios (SUVRs) were obtained by normalizing the cortical SUV with the mean uptake in a reference region. For the present study, subcortical reference regions were the whole cerebellum (for the 3 groups), and the pons and a composite region (ie, the whole cerebellum, pons, and eroded subcortical white matter) for the NT1 and ADNI groups. ${ }^{28}$ Thus, the following SUVRs were used: cerebellar (with the previously validated cutoff of 1.17),,$^{25}$ pontine, and composite SUVR. PET images were also visually assessed by 3 observers and classified as $\mathrm{A} \beta$ positive or negative, as previously described. $^{29}$

\section{CSF Biomarker Assessments}

CSF samples were collected retrospectively in 20 patients with NT1, and aliquots were frozen and stored immediately at $-80^{\circ} \mathrm{C}$ at the Montpellier CSF-Neurobank (\#DC-2008-417 of the certified NFS 96-900 university hospital center resource center BB-0033-00031, www.biobanques.eu). CSF A $\beta_{42}, A \beta_{40}$, total tau, and phosphorylated tau ( $\mathrm{p}$-tau 181) levels were measured using the standardized, commercially available Innotest sandwich enzyme-linked immunosorbent assay (Fujirebio Diagnostics, Malvern, PA), as previously described. ${ }^{30}$ CSF orexin-A level was determined in duplicate using the $\mathrm{I}^{125}$-radioimmunoassay (RIA) kit from Phoenix Pharmaceuticals (Burlingame, CA), according to the manufacturer's recommendations.

\section{Statistical Analysis}

Clinical and neuropsychological data, and amyloid load (based on SUVRs) were compared in the 3 groups (NT1, ADNI, and MAPT-AV45) using the Wilcoxon matched-pairs signed-rank test for continuous variables, the McNemar test for binary data, and the Bowker test of symmetry for categorical data with $>2$ categories. Spearman rank order correlations were used to determine associations between continuous variables. A correction for multiple comparisons for the SUVRs was applied using the false discovery rate procedure. Significance level was set at $p<0.05$. Statistical analyses were performed using SAS, version 9.4 (SAS Institute, Cary, NC).

\section{Results}

\section{Characteristics of the Different Groups}

Among the 23 patients with NT1, 4 (17.4\%) were obese, $14(66.6 \%)$ had persistent sleepiness (ESS > 10), and $19(82.6 \%)$ took psychostimulant medications (mainly modafinil, methylphenidate, and sodium oxybate). Moreover, $17(73.9 \%)$ had a history of cardiovascular disease (mainly hypertension), and $2(8.6 \%)$ reported depressive symptoms (Table 1). Thirteen patients had a cognitive complaint $(56.5 \%)$, including 7 patients with $\mathrm{CDR}=0.5$ and 1 with $\mathrm{CDR}=1$. Based on the neuropsychological evaluation, $21.7 \%$ of patients with NT1 had an episodic memory deficit (ie, hippocampal syndrome), and 60.9\% had an executive dysfunction (see Table 1). The median (range) scores were 29 (15-30) for the MMSE, 16 (12-18) for the FAB, and 35 (25-36) for the ReyOsterrieth Complex Figure Test (with a median time to perform the test of 130 seconds [65-330]). No praxis alteration was observed. The only patient with mild dementia $(C D R=1)$ had a past history of stroke and extrapyramidal signs potentially related to vascular damages (MRI Fasekas score $=3$ ). Family history of dementia 
TABLE 1. Clinical, Neuropsychological, and Biological Characteristics of the 23 Patients with Narcolepsy Type 1

Characteristic

Clinical assessment

Age at onset, yr

Age at diagnosis, yr

23

23

$27.00(11 ; 61)$

BMI

$<25 \mathrm{~kg} / \mathrm{m}^{2}$

5

$25-30 \mathrm{~kg} / \mathrm{m}^{2}$

14

$\geq 30 \mathrm{~kg} / \mathrm{m}^{2}$

ESS total score

$\leq 10$

$11-15$

$\geq 16$

$$
4
$$

21

7

4

10

19

13

17

23

21.7

Psychostimulants drugs, yes

Anticataplectic drugs, yes

Cardiovascular events history, yes ${ }^{\mathrm{a}}$

Beck Depression Inventory total score

Beck Depression Inventory total score

$<12$

12-19

12

9

20-27

$\geq 28$

EQ5D visual analog scale

EQ5D utility

Neuropsychological assessment

Mild cognitive impairment, $\mathrm{CDR}=0.5$

Mild dementia, $\mathrm{CDR}=1$

1

Memory episodic deficit, hippocampal syndrome

5

14

52.2

39.1

1

1

23

23

4.3

4.3

$70(30 ; 95)$

$0.84(0.34 ; 1.00)$

Executive dysfunction

30.4

4.3

21.7

Biology

CSF A $\beta 42$ levels, pg/ml 20

$899.50(300 ; 1,438)$

Abnormal CSF A $\beta 42$ levels, $<500$ pg $/ \mathrm{ml}$

3

15.00

CSF A 340 levels, pg/ml 15

$7,751(2,657 ; 11,759)$

CSF tau levels, pg/ml

20

$193.50(58 ; 407)$

Abnormal CSF tau levels, $>400 \mathrm{pg} / \mathrm{ml}$

1

5.00

CSF p-tau levels, pg/ml

20

$38.50(15 ; 73)$

Abnormal CSF p-tau levels, $>60 \mathrm{pg} / \mathrm{ml}$

10.00

${ }^{a}$ Cardiovascular events are defined as the presence of hypertension, diabetes, myocardial infarction, or stroke.

$\mathrm{BMI}=$ body mass index; $\mathrm{CDR}=$ Clinical Dementia Rating $(0=$ normal; 0.5 = very mild dementia; 1 = mild dementia; 2 = moderate dementia; 3 = severe dementia); CSF = cerebrospinal fluid; EQ5D = European Quality of Life Five Dimensions scale; ESS = Epworth Sleepiness Scale; p-tau = phosphorylated tau. 
was reported by $13.6 \%$ of patients, and adulthood traumatic brain injury by $9 \%$. Apolipoprotein E (APOE) genotyping in 22 patients with NT1 showed that 7 (31.8\%) of them were APOE $\varepsilon 4$ carriers, but none was homozygous for the $\varepsilon 4$ allele. CSF AD biomarker analysis $(n=20$ patients) showed normal CSF p-tau levels in $18(90 \%)$ patients (only 2 with values $>60 \mathrm{pg} / \mathrm{ml}$ : 70 and $73 \mathrm{pg} / \mathrm{ml}$, respectively), and normal A $\beta 42$ concentration $(>500 \mathrm{pg} / \mathrm{ml})$ in $17(85 \%)$ patients $(300,309$, and $438 \mathrm{pg} / \mathrm{ml}$ in the other 3 patients). These 5 patients with $1 \mathrm{AD}$ abnormal biomarker/each did not have episodic memory deficits, and their CDR score was 0 . The median interval between the lumbar puncture and the PET scan was 1.05 years (range $=0-16.49$ ).

Comparison of the 3 groups showed that the MMSE score was lower and CDR scores of $>0$ more frequent in the NT1 than in the ADNI control group. Conversely, no difference was found between the NT1 and the MAPT-AV45 groups (Table 2). No between-group difference was found for the presence of the APOE $€ 4$ allele.

\section{Amyloid Burden in Patients with NT1 and Controls}

The cortical/cerebellum SUVRs were lower in patients with NT1 than in the 2 control groups, when assessed as continuous variables $(p<0.0001$; Table 3, Fig. 1). Similar results were obtained for the cortical/pons and cortical/composite SUVRs between the NT1 and ADNI groups. Similarly, the SUVRs of the 6 cortical regions of interests, regardless of the reference subcortical area, were all lower in the NT1 group than in the ADNI $(p<0.001)$ and MAPT-AV45 groups ( $p<0.05$, but for the cingulum regions).

Using the validated cortical/cerebellum SUVR cutoff of 1.17 , only 1 patient with NT1 (4.3\%) had positive amyloid burden, compared with $27.5 \%$ of the ADNI and $30.4 \%$ of the MAPT-AV45 controls $(p<0.05$; see Table 3). PET image visual analysis (only for patients with NT1 and MAPT-AV45 controls) confirmed that only 1 patient with NT1 had an A $\beta$-positive PET scan compared with $6(26.1 \%)$ MAPT-AV45 controls $(p<0.10$; see Table 3).

\section{TABLE 2. Demographical and Cognitive Characteristics of Patients with NT1 and Controls from the ADNI and MAPT Cohorts}

\begin{tabular}{|c|c|c|c|c|c|c|c|c|}
\hline \multirow[b]{2}{*}{ Characteristic } & \multicolumn{2}{|c|}{$\begin{array}{c}\text { NT1 } \\
\text { Patients, } \\
\text { n }=23\end{array}$} & \multicolumn{2}{|c|}{$\begin{array}{c}\text { ADNI Controls, } \\
n=69\end{array}$} & \multicolumn{2}{|c|}{$\begin{array}{c}\text { MAPT } \\
\text { Controls, } \\
\mathbf{n}=\mathbf{2 3}\end{array}$} & \multirow{2}{*}{$\begin{array}{l}\text { ADNI Controls } \\
\text { vs NT1 } \\
\text { Patients, } p\end{array}$} & \multirow{2}{*}{$\begin{array}{l}\text { MAPT Controls } \\
\text { vs NT1 } \\
\text { Patients, } p\end{array}$} \\
\hline & $\mathbf{n}$ & $\%$ & $\mathbf{n}$ & $\%$ & $\mathbf{n}$ & $\%$ & & \\
\hline \multicolumn{9}{|l|}{ Sex } \\
\hline Male & 14 & 60.9 & 42 & 60.9 & 14 & 60.9 & - & - \\
\hline Female & 9 & 39.1 & 27 & 39.1 & 9 & 39.1 & & \\
\hline \multicolumn{9}{|l|}{ Age, in years } \\
\hline$<75$ & 14 & 60.9 & 42 & 60.9 & 14 & 60.9 & - & - \\
\hline $75-80$ & 4 & 17.4 & 12 & 17.4 & 4 & 17.4 & & \\
\hline$\geq 80$ & 5 & 21.7 & 15 & 21.7 & 5 & 21.7 & & \\
\hline MMSE score ${ }^{a}$ & \multicolumn{2}{|c|}{$29(15 ; 30)$} & \multicolumn{2}{|c|}{$29(25 ; 30)$} & \multicolumn{2}{|c|}{$28(25 ; 30)$} & 0.006 & 0.92 \\
\hline$<26$ & 2 & 8.7 & 1 & 1.5 & 1 & 4.4 & 0.03 & 0.56 \\
\hline$\geq 26$ & 21 & 91.3 & 68 & 98.5 & 22 & 95.6 & & \\
\hline \multicolumn{9}{|l|}{ CDR scale } \\
\hline Normal & 15 & 65.22 & 63 & 91.30 & 12 & 52.17 & 0.0001 & 0.41 \\
\hline Very mild/mild dementia & $7 / 1$ & 34.78 & $6 / 0$ & 8.70 & $11 / 0$ & 47.83 & & \\
\hline \multicolumn{9}{|l|}{ Carrier of the APOE $€ 4$ allele } \\
\hline No & 15 & 68.18 & 48 & 69.57 & 15 & 71.43 & 0.90 & 0.82 \\
\hline Yes & 7 & 31.82 & 21 & 30.43 & 6 & 28.57 & & \\
\hline
\end{tabular}


TABLE 3. Comparisons of Brain Amyloid Burden Measured by ${ }^{18} \mathrm{~F}$-Florbetapir PET between Patients with NT1 and Controls from the ADNI and MAPT-AV45 Cohorts

\begin{tabular}{|c|c|c|c|c|c|}
\hline Variable & $\begin{array}{l}\text { NT1 Patients, } \\
\mathbf{n}=\mathbf{2 3} \\
\text { Mean }( \pm \mathrm{SD})\end{array}$ & $\begin{array}{l}\text { ADNI Controls, } \\
\mathbf{n}=\mathbf{6 9} \\
\text { Mean }( \pm \text { SD })\end{array}$ & $\begin{array}{l}\text { MAPT-AV45 } \\
\text { Controls, } \mathbf{n}=23 \\
\text { Mean }( \pm \mathrm{SD})\end{array}$ & $\begin{array}{l}\text { ADNI vs } \\
\text { NT1, } p\end{array}$ & $\begin{array}{l}\text { MAPT-AV45 } \\
\text { vs NT1, } p\end{array}$ \\
\hline SUVR cortical/cerebellum & $0.95( \pm 0.15)$ & $1.11( \pm 0.18)$ & $1.14( \pm 0.17)$ & $<0.0001$ & 0.0005 \\
\hline SUVR cortical/pons & $0.57( \pm 0.09)$ & $0.68( \pm 0.11)$ & - & $<0.0001$ & \\
\hline SUVR cortical/composite score & $0.66( \pm 0.09)$ & $0.78( \pm 0.11)$ & - & $<0.0001$ & \\
\hline SUVR frontal/cerebellum & $0.92( \pm 0.16)$ & $1.10( \pm 0.20)$ & $1.08( \pm 0.13)$ & $<0.0001$ & 0.0006 \\
\hline SUVR parietal/cerebellum & $0.93( \pm 0.17)$ & $1.08( \pm 0.19)$ & $1.08( \pm 0.16)$ & $<0.0001$ & 0.001 \\
\hline SUVR temporal/cerebellum & $0.98( \pm 0.12)$ & $1.08( \pm 0.16)$ & $1.18( \pm 0.14)$ & $<0.0001$ & 0.0005 \\
\hline SUVR precuneus/cerebellum & $1.05( \pm 0.22)$ & $1.18( \pm 0.23)$ & $1.20( \pm 0.25)$ & 0.0003 & 0.02 \\
\hline SUVR anterior cingulate/cerebellum & $1.13( \pm 0.21)$ & $1.28( \pm 0.23)$ & $1.16( \pm 0.18)$ & $<0.0001$ & 0.93 \\
\hline \multirow[t]{2}{*}{ SUVR posterior cingulate/cerebellum } & $1.10( \pm 0.20)$ & $1.22( \pm 0.23)$ & $1.16( \pm 0.23)$ & 0.0007 & 0.62 \\
\hline & $\%$ & $\%$ & $\%$ & & \\
\hline \multicolumn{6}{|l|}{ SUVR cortical/cerebellum } \\
\hline$\leq 1.17$ & 95.7 & 72.5 & 69.6 & 0.0003 & 0.04 \\
\hline$>1.17$ & 4.3 & 27.5 & 30.4 & & \\
\hline \multicolumn{6}{|l|}{ Positive PET-AV45 visual rating } \\
\hline No & 95.7 & - & 73.9 & - & 0.08 \\
\hline Yes & 4.3 & - & 26.1 & - & \\
\hline
\end{tabular}
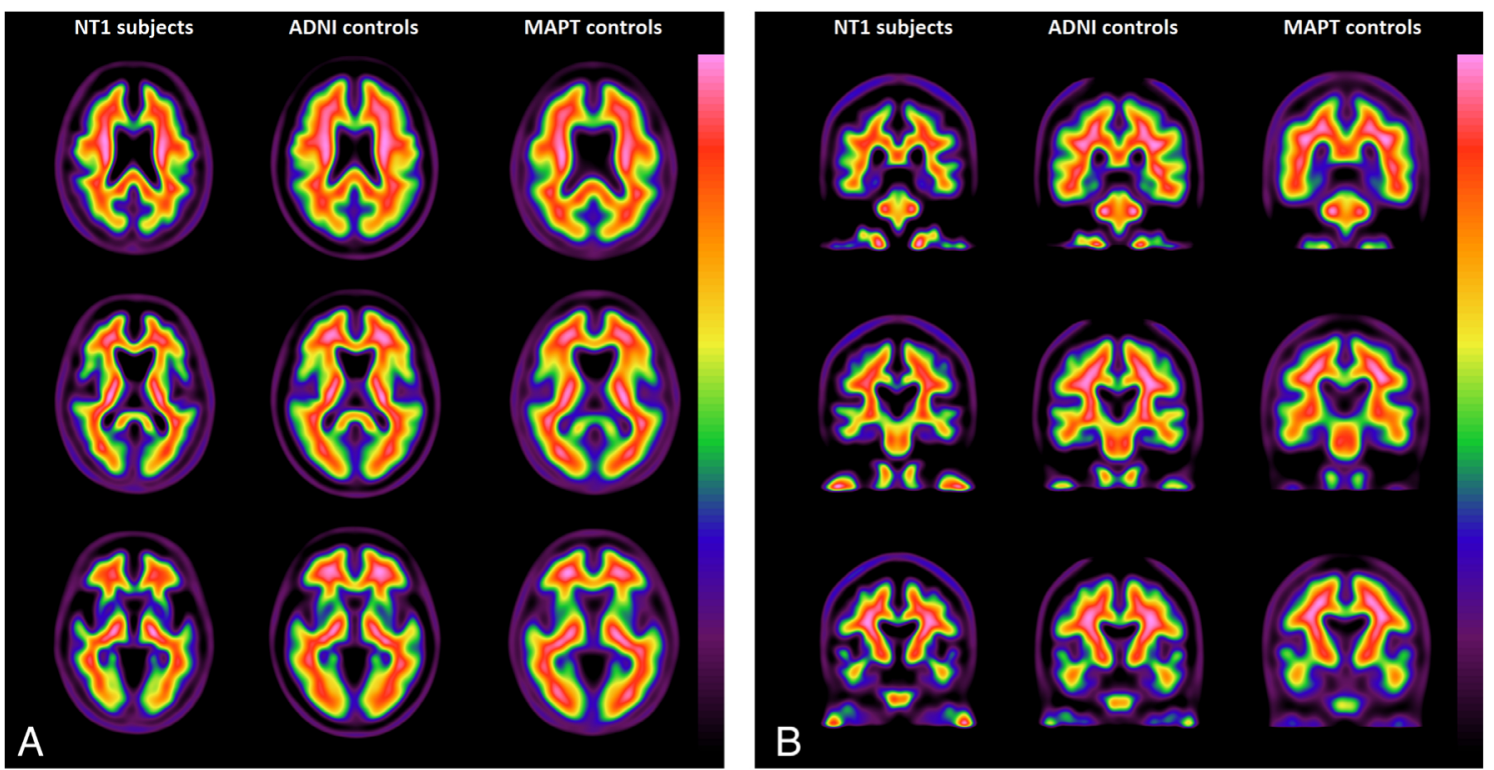

FIGURE 1: Mean ${ }^{18} \mathrm{~F}$-florbetapir tracer uptake in representative (A) axial and (B) coronal slices showing less cortical amyloid load in patients with narcolepsy type 1 (NT1) than in Alzheimer's Disease Neuroimaging Initiative (ADNI) and Multi-Domain Intervention Alzheimer's Prevention Trial (MAPT) controls. 
The only patient with $\mathrm{NT} 1$ and $A \beta$-positive PET was a 68 -year-old woman with late onset of EDS and cataplexy (ie, at 60 years of age), normal cognitive profile (no cognitive complaint, amnesia, aphasia, apraxia, or agnosia), $\mathrm{CDR}=0, \mathrm{MMSE}=30$, and FAB, TMT-A and $\mathrm{B}$, and FCSRT free and total recall scores in the normal range. Her CSF orexin-A level was $19 \mathrm{pg} / \mathrm{ml}$, tau = $148 \mathrm{pg} / \mathrm{ml}, \quad \mathrm{p}$-tau $=41 \mathrm{pg} / \mathrm{ml}, \quad \mathrm{A} \beta_{40}=7,861 \mathrm{pg} / \mathrm{ml}$, and $A \beta_{42}=438 \mathrm{pg} / \mathrm{ml}$. MRI revealed no cortical atrophy and normal hippocampal volume (Scheltens score $=0$, right and left), but some minor white matter hyperintense lesions (Fazekas score $=1)$. Fluorodeoxyglucose PET imaging highlighted a mild left parietal hypometabolism that did not reach the pathological threshold.

A subanalysis after exclusion of all participants with CDR $\geq 0.5$ (ie, 8 patients with NT1, 6 ADNI and 11 MAPT-AV45 controls) confirmed lower cortical/cerebellum SUVRs in the NT1 than in the 2 control groups $(p<0.0006)$. We performed another subanalysis excluding all participants with abnormal CSF A $\beta 42$ concentration from the NT1 and ADNI populations (ie, 3 patients with NT1 and 28 subjects from ADNI; no CSF was available in the MAPT-AV45 population) and confirmed lower cortical/cerebellum SUVRs in the NT1 than in the ADNI group (0.92 \pm 0.10 vs $1.02 \pm 0.10, p=0.0007)$. None of the 6 subjects from the ADNI cohort with a CDR of 0.5 had abnormal CSF A $\beta 42$ levels. The SUVRs were lower in the NT1 than in the 2 control groups for the 6 cortical regions of interest, except for the cingulum regions when comparing the NT1 and MAPT-AV45 groups, and regardless of the subcortical reference area (cerebellum, pons, composite areas) for the comparison between the NT1 and ADNI groups.

Finally, in patients with NT1, no association was found between cortical or regional SUVRs and CSF orexin- $\mathrm{A}$ and $\mathrm{AD}$ biomarkers or neuropsychological assessment results. No correlation was found between NT1 duration and CSF levels of tau, p-tau, and A $\beta 42$.

\section{Discussion}

Using ${ }^{18}$ F-florbetapir PET and the semiquantitative cortical SUVR method, we found lower levels of cortical amyloid burden in elderly patients with NT1 than in 2 age- and sex-matched control groups free of clinical dementia (ADNI and MAPT-AV45). Using the validated cortical/cerebellum SUVR cutoff, only 1 patient with NT1 (4.3\%) had A $\beta$-positive PET compared with $27.5 \%$ of the ADNI and $30.4 \%$ of the MAPT-AV45 controls. Finally, in patients with NT1, we did not find any association between cortical/regional amyloid load and CSF orexin-A levels, CSF A $\beta 42$ or A $\beta 40$ concentration, or neuropsychological profile.
${ }^{18}$ F-Florbetapir PET is a reliable tool to identify subjects at greatest risk of $\mathrm{AD}$, because amyloid plaque deposition begins many years before the detection of cognitive and noncognitive symptoms. ${ }^{31}$ Several clinicalhistopathological studies have highlighted ${ }^{18} \mathrm{~F}$-florbetapir PET high affinity and specificity for $A \beta$ and the correlation with the amyloid burden at autopsy. ${ }^{31}$ The low levels of cortical A $\beta$ in elderly patients with NT1 suggest a reduced risk for progression to AD. The only patient with NT1 and $A \beta$-positive PET was remarkable due to the late disease onset ( 60 years of age when normally it is before the age of 30 years) and the normality of her neuropsychological profile. Conversely, among the ADNI and MAPT-AV45 controls, $27.5 \%$ and $30.4 \%$, respectively, had $A \beta$-positive PET. These results are in agreement with most studies on healthy controls. ${ }^{32,33}$ More than half of patients with NT1 reported cognitive complaints, in agreement with the literature. $^{34}$ We diagnosed mild dementia $(\mathrm{CDR}=1)$ in 1 patient with NT1 and normal amyloid PET, in the context of cerebrovascular and Parkinson disease.

For this study, we used both a manual visual and a semiquantitative (SUVR) approach to evaluate $A \beta$ load and then compare the 3 groups. For the semiquantitative approach, we focused on 6 cortical regions and used 3 reference subcortical regions (cerebellum, pons, and the most sensitive composite areas), when possible, and the validated cortical/cerebellum SUVR cutoff of 1.17. ${ }^{25,29}$ We obtained similar results (ie, lower amyloid load in NT1, except for the cingulum between the NT1 and MAPT-AV45 groups) with the different approaches, although the manual visual analysis is a less accurate method. ${ }^{29}$ As $A \beta$ load could be influenced by cognitive status alterations, we performed additional analyses after exclusion of all people with a CDR score $\geq 0.5$ to retain only people with normal cognitive status and confirmed the previous results. We also confirmed lower amyloid load in NT1 than in the ADNI group after exclusion of all participants with abnormal CSF A $\beta 42$ levels (ie, data available in the NT1 and ADNI populations).

To our knowledge, brain amyloid deposition has never been quantified by PET in patients with NT1. However, there are some data on postmortem neuropathological $\mathrm{AD}$ lesions and CSF concentration of $\mathrm{AD}$ biomarkers in narcolepsy. The only study with postmortem neuropathological confirmation evaluated a 75-year-old man with narcolepsy-cataplexy since the age of 12 years, and cognitive complaint for 8 years. ${ }^{18}$ Postmortem histological analysis showed loss of $85 \%$ of orexinergic neurons and extensive plaques and tangles in targeted regions, consistent with AD. The author extended the analysis to another 11 patients with narcolepsy-cataplexy and found that 33\% had $\mathrm{AD}$ lesions (same prevalence as in the general population). However, in these additional patients, orexinergic 
neurons and CSF orexin-A levels were not quantified, the clinical records of narcolepsy and cognitive profiles were poorly reported, the cause of the autopsy request and related selection bias were missing, and the mean age at death was very old $(81$ years, range $=75-94) .{ }^{18}$ Some differences between this work and the present study (ie, detailed phenotype, orexin-A status, age at evaluation, postmortem neuropathology vs in vivo analysis) could explain the different findings.

Previous studies assessed CSF A $\beta$ levels in small groups of patients with narcolepsy. Discrepancies between their results ${ }^{11,35-39}$ can be partially explained by differences in the populations under study (age, disease duration, type 1 vs type 2 narcolepsy, drug-free or not, and choice of controls), the sample size, and also the method used for orexin-A quantification (RIA vs enzyme immunoassays). Some studies reported lower levels of CSF A $\beta 42$ in patients with NT1 and also in patients with NT2 (ie, who have normal orexin levels), ${ }^{37,39}$ especially in younger patients and those with short disease duration. Conversely, other workers found normal or even increased CSF A $\beta 42$ levels ${ }^{11}$ in patients with NT1 compared with controls, especially in patients with longer disease duration and with stable stimulant intake. ${ }^{40}$ In our study, the long disease duration (median age at onset $=27$ years, median age at time of study $=71$ years) and the high frequency of psychostimulant intake in the NT1 group could explain the absence of significant changes in CSF A $\beta$ levels (ie, normal CSF $A \beta 42$ levels in $17 / 20$ patients with NT1). Analysis of CSF $A \beta 42$ and orexin levels in patients with $\mathrm{AD}$ has also led to discordant results. ${ }^{11,41} \mathrm{We}$ found higher CSF orexin-A levels, but not histamine, in patients with early and advanced $\mathrm{AD}$ than in those with other dementia types, with a negative correlation between $A \beta_{42}$ and orexin- $\mathrm{A}$ in $\mathrm{AD} .{ }^{42}$ Similarly, other studies reported increased CSF orexin-A levels in patients with $\mathrm{AD}$ at different stages of disease progression. ${ }^{10,11}$ Conversely, 2 other studies showed normal or low CSF orexin-A levels in AD. ${ }^{10,43}$ Again, these discrepancies could be explained by differences in methodology, population characteristics, small sample sizes, and the influence of uncontrolled factors, such as sleep-wake states. Altogether, these different findings suggest that orexin- $\mathrm{A}$ might influence amyloid clearance and aggregation in the brain. However, other factors could also be involved, such as immunological or neuroinflammatory mechanisms, neuronal activity levels, changes in the glymphatic pathway, and sleep-wake cycle abnormalities.

In the present study, CSF A $\beta 42$ levels were abnormal in 3 patients with NT1, including the one with positive ${ }^{18}$ F-florbetapir PET. However, the neuropsychological test scores were normal in all of them (no hippocampal syndrome and $\mathrm{CDR}=0$ ), as well as the MRI, and CSF tau and p-tau profiles. Although CSF and PET-based A $\beta$ measurement are highly correlated in the literature, some discordances may exist, especially in cognitively normal participants, where 15 to $20 \%$ of people have low CSF A $\beta$ and normal amyloid PET. ${ }^{44}$ In contrast to PET with tracers that bind to amyloid plaques, the CSF concentration of soluble $A \beta$ could reflect different aspects of $A D$ pathology and be influenced by neuroinflammation and sleepwake patterns. Recent studies confirmed that reduced CSF A $\beta 42$ level is an earlier biomarker of $\mathrm{AD}$ compared with amyloid $\mathrm{PET}^{45}$; however, semiquantitative PET assessment is more powerful for accurate grading of early stage $\mathrm{AD}$ and AD conversion prognosis. ${ }^{46}$ The mechanism underlying abnormal $A \beta$ metabolism in NT1 remains unclear, but orexin deficiency, neuroinflammation, and abnormal sleepwake patterns could be involved in brain amyloidosis. Although normal orexin-A levels are certainly not a prerequisite for $\mathrm{AD}$ pathogenesis, we could hypothesize that longterm loss of orexin signaling affects the balance between $A \beta$ production and degradation/clearance and that in patients with NT1 the risk of developing AD could be reduced or the appearance of amyloid plaques and related disease symptoms delayed.

The present study has some strengths, particularly the well-defined phenotype of patients with NT1, all with clear-cut cataplexy (CSF orexin-A measurements for $87 \%$ of them to confirm the diagnosis) and a comprehensive neuropsychological evaluation. We assessed amyloid pathology by measuring CSF $A \beta 42$ and $A \beta 40$ levels and also by using brain ${ }^{18} \mathrm{~F}$-florbetapir PET. The method used to analyze the ${ }^{18} \mathrm{~F}$-florbetapir PET images was the same for the 3 groups (NT1, ADNI, and MAPT-AV45), and included the most sensitive and reliable quantification assessment to increase the diagnostic performance.

Some limitations also need to be acknowledged, particularly the limited number of patients with NT1. However, NT1 is an orphan disease and only subjects older than 65 years were included. As concurrent MRI brain imaging data were available for only 4 patients with NT1, with long time intervals between the MRI and the PET data acquisition (median interval $=1,581$ days, range $=$ 125-3,089 days), PET amyloid in the NT1 group was calculated after spatial normalization with the standard MNI data from the MRI template, whereas the PET amyloid in the control groups was calculated using the individual structural MRI, leading to a potential limitation bias. However, the analysis limited to the SUVR of the 4 NT1 patients with concurrent MRI data showed no difference between the results obtained after normalization using the MRI generic template and the MRI individual data. Similarly, the analysis in the $69 \mathrm{ADNI}$ participants using the generic MRI template for normalization (as for the patients with 
NT1) did not reveal any difference from the SUVRs obtained using their MRI individual data (data not shown). Regarding the strong association reported between NT1 and HLA DQB $1 * 06: 02$, and the evidence supporting the role of neuroinflammation in AD pathophysiology, we cannot exclude that this genotype might influence both amyloid metabolism and orexin-A levels via immunological or neuroinflammatory mechanisms. However, the limited data on HLA typing available in the ADNI group $(<40 \%)$ and their absence in the MAPT-AV45 group did not allow us to control our results for HLA. The 2 control groups included subjects free of clinical dementia at the time of study, but we cannot exclude that some of them may later develop AD or other dementias. However, subanalysis after exclusion of participants with CDR $\geq 0.5$ confirmed lower cortical/cerebellum SUVRs in the NT1 group than in the 2 control groups. In the same way, exclusion of participants with abnormal CSF A $\beta 42$ concentration from both the ADNI and NT1 populations confirmed lower cortical/cerebellum SUVRs in the NT1 group. None of the subjects from the ADNI cohort with a CDR of $0.5(n=6)$ had abnormal CSF A $\beta 42$ levels. No differences in the median of CSF A 342 levels were found between subjects with CDR of 0 and those with CDR of 0.5 in the ADNI population. No CSF orexin-A levels were available for either control group, and no CSF AD biomarkers were available for the MAPT-AV45 population. The CSF procedure for patients with NT1 was chosen for a diagnostic purpose, to measure the concentration of orexin-A to validate the diagnosis of NT1. We did not perform a second lumbar puncture for this specific study. The variable interval between PET data acquisition and CSF sampling in the NT1 group remains an additional limitation that could explain the lack of correlation between AD markers and NT1 duration. Finally, we were unable to include a population of age- and sex-matched patients with $\mathrm{AD}$ with available PET with ${ }^{18}$ F-florbetapir. However, the inclusion of such a group of patients with $\mathrm{AD}$, with a range of 61 to $84.7 \%$ PET amyloid positive for diagnosed $\mathrm{AD}$ patients (ie, diagnosis made with clinical signs only or with both positive clinical signs and biomarkers, respectively), ${ }^{47,48}$ would have led to an even more significant difference from patients with NT1 $(p<0.0001)$ than with the other control populations free of clinical dementia.

Our results highlight a lower brain $\mathrm{A} \beta$ burden, detected by ${ }^{18} \mathrm{~F}$-florbetapir PET, in elderly patients with orexin-A-deficient narcolepsy, suggesting a lower risk of amyloidopathy related to AD in NT1. Longitudinal amyloid PET and cognitive studies in elderly patients with NT1 are required to confirm this result and to understand its implications. For instance, this could lead to reconsidering the potential protective effect of orexin receptor antagonists on brain amyloid load in subjects at high risk of $\mathrm{AD}$.

\section{Acknowledgment}

This study was supported by the PROTECMAN internal call for tenders of the Montpellier University Hospital Center (ClinicalTrials.gov identifier NCT03378453). The MAPT-AV45 study was supported by grants from the Gérontopôle of Toulouse, the French Ministry of Health (PHRC 2008, 2009), the Pierre Fabre Research Institute (manufacturer of the omega-3 supplement), ExonHit Therapeutics, and Avid Radiopharmaceuticals. The promotion of this study was supported by the University Hospital Center of Toulouse. Data sharing was supported by the Monaco Association for Research on Alzheimer's Disease and the National Institute of Health and Medical Research Mixed Unit of Research 1027-University of Toulouse III.

We thank Dr V. Damotte for his expert technical assistance.

\section{Author Contributions}

Y.D. and A.G. contributed to the conception and design of the study; all authors contributed to the acquisition and analysis of data; Y.D., A.G., F.B.B., I.J., R.L., and L.B. contributed to drafting the text and preparing the figure.

\section{Potential Conflicts of Interest}

Nothing to report.

\section{References}

1. Frisoni GB, Lorenzi M, Caroli $A$, et al. In vivo mapping of amyloid toxicity in Alzheimer disease. Neurology 2009;72:1504-1511.

2. Jack CR Jr, Knopman DS, Jagust WJ, et al. Hypothetical model of dynamic biomarkers of the Alzheimer's pathological cascade. Lancet Neurology 2010;9:119-128.

3. Bateman RJ, Xiong C, Benzinger TL, et al. Clinical and biomarker changes in dominantly inherited Alzheimer's disease. N Engl J Med 2012;367:795-804

4. McDade E, Bateman RJ. Stop Alzheimer's before it starts. Nature 2017;547:153-155.

5. Peng W, Achariyar TM, Li B, et al. Suppression of glymphatic fluid transport in a mouse model of Alzheimer's disease. Neurobiol Dis 2016;93:215-225

6. Jessen NA, Munk AS, Lundgaard I, Nedergaard M. The glymphatic system: a beginner's guide. Neurochem Res 2015;40:2583-2599.

7. Tarasoff-Conway JM, Carare RO, Osorio RS, et al. Clearance systems in the brain-implications for Alzheimer disease. Nat Rev Neurol 2015;11:457-470.

8. Kang JE, Lim MM, Bateman RJ, et al. Amyloid-beta dynamics are regulated by orexin and the sleep-wake cycle. Science 2009;326: 1005-1007. 
9. Roh $\mathrm{JH}$, Jiang $\mathrm{H}$, Finn $M B$, et al. Potential role of orexin and sleep modulation in the pathogenesis of Alzheimer's disease. J Exp Med 2014;211:2487-2496.

10. Fronczek R, van Geest S, Frolich M, et al. Hypocretin (orexin) loss in Alzheimer's disease. Neurobiol Aging 2012;33:1642-1650.

11. Dauvilliers YA, Lehmann S, Jaussent I, Gabelle A. Hypocretin and brain beta-amyloid peptide interactions in cognitive disorders and narcolepsy. Front Aging Neurosci 2014;6:119.

12. Wennstrom M, Londos $E$, Minthon L, Nielsen HM. Altered CSF orexin and alpha-synuclein levels in dementia patients. J Alzheimers Dis 2012;29:125-132

13. Liguori C, Romigi A, Nuccetelli M, et al. Orexinergic system dysregulation, sleep impairment, and cognitive decline in Alzheimer disease. JAMA Neurol 2014;71:1498-1505.

14. Ju YS, Ooms SJ, Sutphen C, et al. Slow wave sleep disruption increases cerebrospinal fluid amyloid-beta levels. Brain 2017;140: 2104-2111.

15. Shan L, Dauvilliers Y, Siegel JM. Interactions of the histamine and hypocretin systems in CNS disorders. Nat Rev Neurol 2015;11: 401-413.

16. Scammell TE. Narcolepsy. N Engl J Med 2015;373:2654-2662.

17. Dauvilliers $Y$, Montplaisir J, Molinari N, et al. Age at onset of narcolepsy in two large populations of patients in France and Quebec. Neurology 2001;57:2029-2033.

18. Scammell TE, Matheson JK, Honda M, et al. Coexistence of narcolepsy and Alzheimer's disease. Neurobiol Aging 2012;33:1318-1319.

19. American Academy of Sleep Medicine. International classification of sleep disorders-third edition (ICSD-3). Darien, IL: American Academy of Sleep Medicine, 2014.

20. Johns MW. A new method for measuring daytime sleepiness: the Epworth Sleepiness Scale. Sleep 1991;14:540-545.

21. Beck $A T$, Ward $C H$, Mendelson $M$, et al. An inventory for measuring depression. Arch Gen Psychiatry 1961;4:561-571

22. Rabin R, de Charro F. EQ-5D: a measure of health status from the EuroQol Group. Ann Med 2001;33:337-343.

23. Hughes $\mathrm{CP}$, Berg L, Danziger WL, et al. A new clinical scale for the staging of dementia. Br J Psychiatry 1982;140:566-572.

24. Saykin AJ, Wishart HA, Rabin LA, et al. Older adults with cognitive complaints show brain atrophy similar to that of amnestic $\mathrm{MCl}$. Neurology 2006;67:834-842.

25. Vellas B, Carrie I, Gillette-Guyonnet S, et al. MAPT study: a multidomain approach for preventing Alzheimer's disease: design and baseline data. J Prev Alzheimers Dis 2014;1:13-22.

26. Joshi AD, Pontecorvo MJ, Clark CM, et al. Performance characteristics of amyloid PET with florbetapir F 18 in patients with Alzheimer's disease and cognitively normal subjects. J Nucl Med 2012;53: 378-384.

27. Joshi AD, Koeppe RA, Fessler JA, Kilbourn MR. Signal separation and parameter estimation in noninvasive dual-tracer PET scans using reference-region approaches. J Cereb Blood Flow Metab 2009;29: $1346-1357$

28. Schreiber S, Landau SM, Fero A, et al. Comparison of visual and quantitative florbetapir $\mathrm{F} 18$ positron emission tomography analysis in predicting mild cognitive impairment outcomes. JAMA Neurol 2015;72:1183-1190.

29. Payoux P, Delrieu J, Gallini A, et al. Cognitive and functional patterns of nondemented subjects with equivocal visual amyloid PET findings. Eur J Nucl Med Mol Imaging 2015;42:1459-1468.
30. Dumurgier J, Vercruysse $\mathrm{O}$, Paquet $\mathrm{C}$, et al. Intersite variability of CSF Alzheimer's disease biomarkers in clinical setting. Alzheimers Dement 2013;9:406-413.

31. Clark CM, Schneider JA, Bedell BJ, et al. Use of florbetapir-PET for imaging beta-amyloid pathology. JAMA 2011;305:275-283.

32. Pontecorvo MJ, Mintun MA. PET amyloid imaging as a tool for early diagnosis and identifying patients at risk for progression to Alzheimer's disease. Alzheimers Res Ther 2011;3:11.

33. Aizenstein HJ, Nebes RD, Saxton JA, et al. Frequent amyloid deposition without significant cognitive impairment among the elderly. Arch Neurol 2008;65:1509-1517.

34. Bayard S, Croisier Langenier M, Cochen De Cock V, et al. Executive control of attention in narcolepsy. PLoS One 2012;7:e33525.

35. Heier MS, Skinningsrud A, Paus E, Gautvik KM. Increased cerebrospinal fluid levels of nerve cell biomarkers in narcolepsy with cataplexy. Sleep Med 2014;15:614-618.

36. Kallweit U, Hidalgo $H$, Engel $A$, et al. Post $H 1 N 1$ vaccination narcolepsy-cataplexy with decreased CSF beta-amyloid. Sleep Med 2012;13:323.

37. Liguori C, Placidi F, Albanese M, et al. CSF beta-amyloid levels are altered in narcolepsy: a link with the inflammatory hypothesis? J Sleep Res 2014;23:420-424

38. Liguori C, Placidi F, Izzi F, et al. Beta-amyloid and phosphorylated tau metabolism changes in narcolepsy over time. Sleep Breath 2016 ; 20:277-283; discussion 283

39. Jørgen Jennum $P$, Østergaard Pedersen L, Czarna Bahl JM, et al. Cerebrospinal fluid biomarkers of neurodegeneration are decreased or normal in narcolepsy. Sleep 2017;40(1).

40. Liguori C, Placidi F, Izzi F, et al. May CSF beta-amyloid and tau proteins levels be influenced by long treatment duration and stable medication in narcolepsy? Sleep Med 2014;15:1424.

41. Slats D, Claassen JA, Verbeek MM, Overeem S. Reciprocal interactions between sleep, circadian rhythms and Alzheimer's disease: focus on the role of hypocretin and melatonin. Ageing Res Rev 2013:12:188-200.

42. Gabelle A, Jaussent I, Hirtz C, et al. Cerebrospinal fluid levels of orexin-A and histamine, and sleep profile within the Alzheimer process. Neurobiol Aging 2017;53:59-66.

43. Schmidt FM, Kratzsch J, Gertz HJ, et al. Cerebrospinal fluid melaninconcentrating hormone $(\mathrm{MCH})$ and hypocretin-1 (HCRT-1, orexin-A) in Alzheimer's disease. PLoS One 2013;8:e63136.

44. Mattsson N, Insel PS, Donohue $M$, et al. Independent information from cerebrospinal fluid amyloid-beta and florbetapir imaging in Alzheimer's disease. Brain 2015;138(pt 3):772-783.

45. Palmqvist S, Mattsson N, Hansson $O$. Cerebrospinal fluid analysis detects cerebral amyloid-beta accumulation earlier than positron emission tomography. Brain 2016;139(pt 4):1226-1236.

46. Doraiswamy PM, Sperling RA, Johnson K, et al. Florbetapir F 18 amyloid PET and 36-month cognitive decline: a prospective multicenter study. Mol Psychiatry 2014;19:1044-1051.

47. Sevigny J, Suhy J, Chiao P, et al. Amyloid PET screening for enrichment of early-stage Alzheimer disease clinical trials: experience in a phase 1b clinical trial. Alzheimer Dis Assoc Disord 2016;30:1-7.

48. Landau SM, Horng A, Fero A et al. Amyloid negativity in patients with clinically diagnosed Alzheimer disease and $\mathrm{MCl}$. Neurology 2016:86:1377-1385. 